\title{
PLASMA RICO EM FIBRINA (PRF) COMO OPÇÃO DE RECOBRIMENTO APÓS ROTAÇÃO DE RETALHO PALATINO PARA TRATAMENTO DE FÍSTULA BUCOSSINUSAL
}

\author{
FIBRIN-RICH PLASMA COVER AS A OPTION AFTER PALATAL FLAP \\ ROTATION FOR TREATMENT OF OROANTRAL FISTULA
}

\section{Rafael Mérola Corrêa}

Residente de Cirurgia Oral e Maxilofacial do Hospital Federal dos Servidores do Estado do Rio de Janeiro - HFSE e da Universidade Federal Fluminense - UFF.

\section{Eugênio Rodrigues Arantes}

Residente de Cirurgia Oral e Maxilofacial do Hospital Federal dos Servidores do Estado do Rio de Janeiro - HFSE e da Universidade Federal Fluminense - UFF.

\section{Ian Magalhães de Andrade}

Residente de Cirurgia Oral e Maxilofacial do Hospital Federal dos Servidores do Estado do Rio de Janeiro - HFSE e da Universidade Federal Fluminense - UFF.

\section{Flávio Merly}

Staff do Serviço de Cirurgia Oral e Maxilofacial do Hospital Federal dos Servidores do Estado do Rio de Janeiro - HFSE e Professor da Faculdade de Odontologia da Universidade Federal Fluminense - UFF.

Rafael

Seabra

Louro

Coordenador do Programa de Residência em Cirurgia Oral e Maxilofacial do Hospital Federal dos Servidores do Estado do Rio de Janeiro - HFSE e da Universidade Federal Fluminense - UFF.

Instituição na qual o trabalho foi realizado: Hospital Federal dos Servidores do Estado do Rio de Janeiro/RJ

Categoria: Relato de caso

Informações do autor principal:

Rafael Mérola Corrêa

Avenida Hildebrando de Araújo Góes, 55, Bloco 01 - Apt 104.

Barra da Tijuca - CEP 22793-250, Rio de Janeiro - RJ

E-mail: rafael.merola@outlook.com 


\section{Resumo}

O desenvolvimento de uma fístula bucossinusal, a partir de uma comunicação gerada entre a cavidade oral e o seio maxilar, pode ser favorecido por um não fechamento espontâneo ou por uma sinusite maxilar crônica já instalada. O tratamento da fístula bucossinusal depende de fatores que podem beneficiar ou dificultar o prognóstico dos casos, como a localização, causa e o tamanho do defeito gerado. Diferentes técnicas são descritas na literatura para o tratamento destes defeitos orosinusais, como retalhos locais deslizantes ou rotacionados, enxertos autógenos ou o uso da bola de gordura bucal. O objetivo do presente trabalho é apresentar o caso clínico de uma fístula bucossinusal pós exodontia há aproximadamente 05 anos, não corrigida imediatamente, tratada através de retalho mucoso palatino rotacionado. Como coadjuvante para a cicatrização da área doadora foi utilizado membrana de fibrina rica em plaquetas. A técnica do retalho palatino mostrou-se uma opção favorável para o fechamento da fístula bucossinusal e o uso dos fatores de crescimento permitiu vascularização adequada na área doadora e no retalho rotacionado, o que favoreceu a hemostasia vascular e estimulou a recuperação dos tecidos moles adjacentes.

Palavras-chave: Seio maxilar, fístula bucoantral, cirurgia bucal, fibrina rica em plaquetas. 


\section{Abstract}

The development of a bucosinusal fistula from a communication generated between the oral and maxillary cavity may be favored by a spontaneous unblocked or an existing chronic maxillary sinusitis. The treatment of bucosinusal fistula depends on factors that may benefit or hinder the prognosis of cases, such as location, cause and size of the defect used. Different techniques are described in the literature for the treatment of these oral defects, such as sliding or rotating local flaps, autogenous grafts or the use of the buccal fat ball. The aim of the present study is to present the clinical case of a postextraction bucosinusal fistula approximately 05 years ago, not immediately corrected, treated through a rotated palatal mucosal flap. As an adjunct to the healing of the donor area, a platelet-rich fibrin membrane was used. The palatal flap technique proved to be a favorable option for the closure of the bucosinusal fistula and the use of growth factors allowed adequate vascularization in the donor area and in the rotated flap, which favored vascular hemostasis and stimulated the recovery of adjacent soft tissues.

Key-words: Maxillary sinus, oroantral fistula, oral surgery, platelet-rich fibrin 


\section{Introdução}

Uma fístula bucossinusal de pequeno diâmetro pode ser fechada imediata e efetivamente no mesmo instante que a comunicação for diagnosticada. A comunicação direta entre a cavidade oral e o seio maxilar pode se desenvolver como uma complicação durante a extração de dentes superiores posteriores devido à proximidade dos ápices destes dentes com a mucosa sinusal (PARVINI et al., 2018). Para o tratamento da fístula bucossinusal deve-se levar em consideração o tamanho do defeito gerado pela comunicação, a presença de infecção maxilar e o tempo de evolução (ABUABARA et al, 2006)

O fechamento de uma fístula bucossinusal pode ser realizado por diferentes técnicas descritas na literatura (PARVINI P. et al, 2018). Algumas estratégias de tratamento são enxertos autógenos ósseos e de tecido mole, materiais alógenos, xenoenxertos, materiais sintéticos entre outras (VISSCHER; VAN MINNEN; BOS, 2010). Para o fechamento de fístulas menores que aproximadamente 1-3 mm de diâmetro, a aproximação primária das bordas reavivadas da fístula pode ser suficiente ou um retalho deslizante do fundo de vestíbulo gengival. Para fístulas maiores que 3 $\mathrm{mm}$, outros métodos de tratamento são indicados como o retalho rotacionado do palato, o uso de enxertos autógenos de tecidos moles como o corpo adiposo bucal ou substâncias e materiais alogênicos (VISSCHER; VAN MINNEN; BOS, 2010).

A técnica de retalho palatino rotacionado é realizada a partir da liberação do pedículo de tecido mole de espessura total, localizado na mucosa que reveste o palato duro, incluindo a artéria palatina maior, responsável pelo suprimento sanguíneo adequado do retalho. A área desnudada do palato será reparada por segunda intenção, sendo sugerida a cobertura com algum tipo de curativo (de Souza Lopes et al, 2015). O retalho palatino apresenta algumas vantagens como não reduzir a profundidade vestibular e por manter sua vascularização própria, porém, a necessidade de deixar uma área desnuda no local doador é considerada sua principal desvantagem (VYLOPPILLI et al, 2019). 
Como opção de tratamento para o fechamento de fístulas bucossinusais, a fibrina rica em plaquetas (PRF) pode ser utilizada por ser um método fácil, rapidamente preparado e implementado, altamente biocompatível e que não apresenta risco de infecção (PARVINI et al, 2018). O PRF tem propriedades que suprimem a inflamação e a infecção, diminui o sangramento local com boa função de hemostasia vascular e estimula a recuperação dos tecidos moles e duros. Assim, a literatura já mostrou a utilização da membrana de fibrina rica em plaquetas concomitantemente com os retalhos bucais e como fechamento direto do defeito sinusal (KAPUSTECKI et al, 2016).

Dessa forma, o objetivo deste trabalho é relatar o caso clínico de uma fístula bucossinusal tratada através do retalho palatino mucoso associado à fibrina rica em plaquetas para o recobrimento da área cruenta no palato duro.

\section{Considerações éticas}

Este trabalho foi escrito foi escrito seguindo as Diretrizes do Relatório de Caso Clínico (CARE, www.care-statement.org) e os padrões éticos propostos pela Declaração de Helsinque de 1975, revisada em 2000.

A paciente foi tratada no Hospital Federal dos Servidores do Estado do Rio de Janeiro conveniado com a Universidade Federal Fluminense através da Residência em Cirurgia Oral e Maxilofacial com os termos, de consentimento livre e esclarecido e de uso de imagens e dados para publicação científica, assinados.

\section{Relato de caso}

Paciente M.C.A.A, 29 anos de idade, sexo feminino, procurou o Serviço de Cirurgia Oral e Maxilofacial do Hospital Federal dos Servidores do Estado do Rio de Janeiro com queixa principal de "dor de cabeça e secreção de gosto ruim na boca" com tempo de evolução de 05 anos após extração dentária. Ao exame físico, apresentava cefaleia intensa e parageusia. Foi observada fístula bucossinusal durante avaliação intraoral com presença de drenagem purulenta na região do elemento dentário \#26 ausente (Figura 1 - A e B). A mesma havia sido submetida a uma tentativa de fechamento da fístula bucossinusal através de retalho vestibular da mucosa oral, porém o tratamento não foi efetivo. Nenhum histórico médico significativo foi relatado. 
Através da associação das informações obtidas na anamnese em conjunto com a avaliação dos exames de imagens de face, chegou-se ao diagnóstico de sinusite maxilar crônica associada a fístula bucossinusal em rebordo alveolar (Figura 1 - C e D). A partir desse momento, teve início o tratamento clínico do seio maxilar esquerdo infectado para obter-se a condição adequada para o procedimento cirúrgico proposto. Foi realizada irrigação do seio maxilar esquerdo com solução de Cloreto de Sódio 0,9\% (Eurofarma Laboratórios S.A. - São Paulo, Brasil) estéril através da fístula bucossinusal três vezes ao dia terapia com Budesonida® ${ }^{\circledR} 50 \mathrm{mcg}$ (Biosintética Farmacêutica - São Paulo, Brasil), na posologia de 2 borrifadas em cada narina 2 vezes ao dia e Penetro Inalante ${ }^{\circledR}$ (Laboratório Daudt de Oliveira LTDA - Rio de Janeiro, Brasil) 3 vezes ao dia. Além disso, foi prescrita Amoxicilina de $875 \mathrm{mg}$ associada ao Clavulanato de Potássio de 125mg (Clavulin® - SmithKline Beecham Pharmaceuticals LTD, Worthing - Inglaterra), na posologia de 12 em 12 horas durante 10 dias.

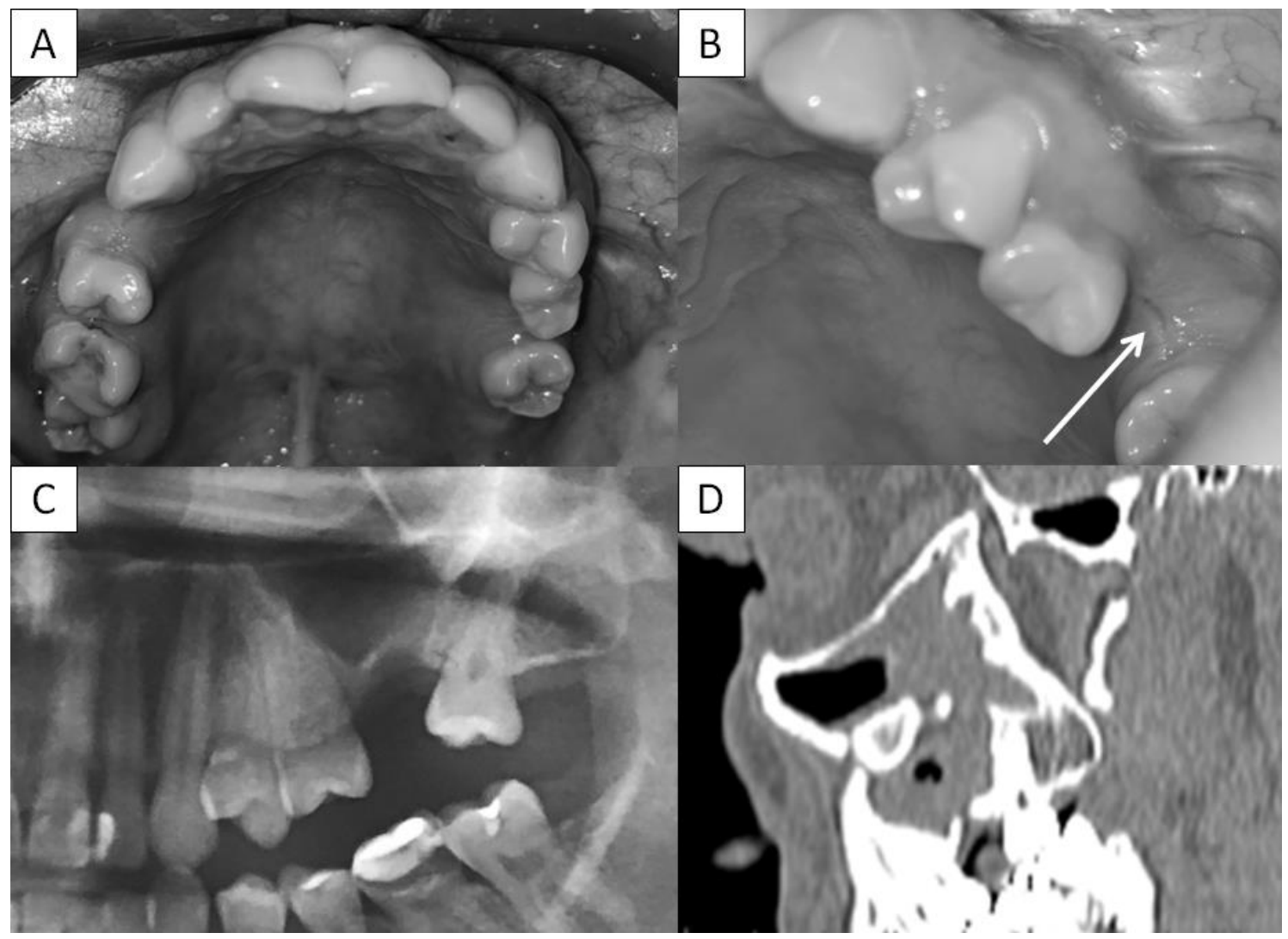

Figura 1: A- Aspecto intraoral geral; B- Imagem correspondente ao fundo de vestíbulo superior esquerdo evidenciando a fístula orosinusal (em seta); C- Radiografia panorâmica demonstrando o álveolo após extração dentária; D- Corte tomográfico sagital com detalhes da comuniação oroantral. 
Após o protocolo citado acima, a paciente estava apta a ser submetida ao novo tratamento cirúrgico para o fechamento da fístula. O procedimento escolhido foi ressecção da fístula bucossinusal associada a retalho palatino rotacionado com membranas de plasma rico em fibrina (PRF) (Figura 2) para o recobrimento da área cruenta do palato e para obliteração da comunicação óssea feita entre o seio maxilar e a cavidade oral. A cirurgia foi realizada, sob anestesia geral, em conjunto com a equipe de Otorrinolaringologia.

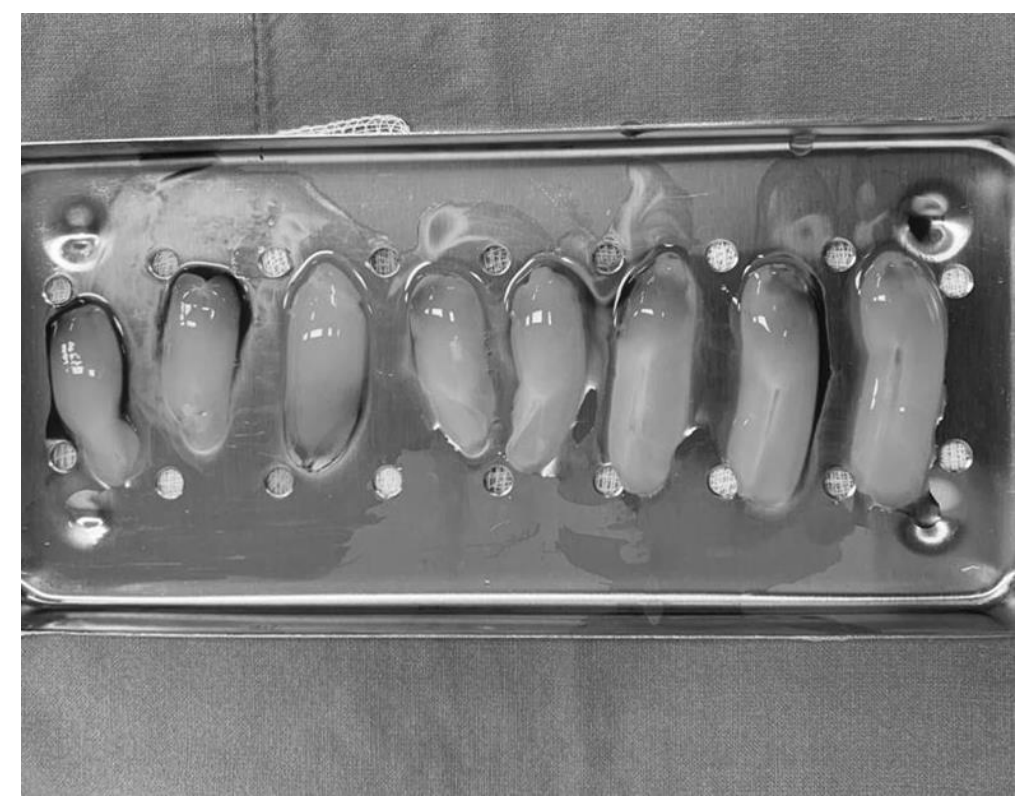

Figura 2: Membranas de plasma rico em fibrina (PRF) após coleta direta da veia mediana cubital e centrifugação em protocolo previamente definido.

O primeiro passo cirúrgico, realizado pelo Serviço de Otorrinolaringologia, foi a antrostomia maxilar com auxílio de vídeo com o objetivo de se obter uma nova via de drenagem do seio maxilar, uma vez que o óstio maxilar encontrava-se completamente obstruído. Finalizada essa etapa, foi realizada a técnica de retalho rotacionado do palato. Esse tecido, de espessura total, foi descolado e sobreposto à fistula bucossinusal para realizar o seu fechamento através de suturas por toda a sua extensão. Para auxiliar no processo de cicatrização da área doadora, foram posicionadas membranas de PRF sobre à área desnuda do palato. Por fim, foi utilizada uma placa de acetato previamente confeccionada através de moldagem da paciente, para proteção tanto da área doadora quanto da receptora.

REVISTA FLUMINENSE DE ODONTOLOGIA - ANO XXVI - No 54 - Julho / Dezembro 2020 


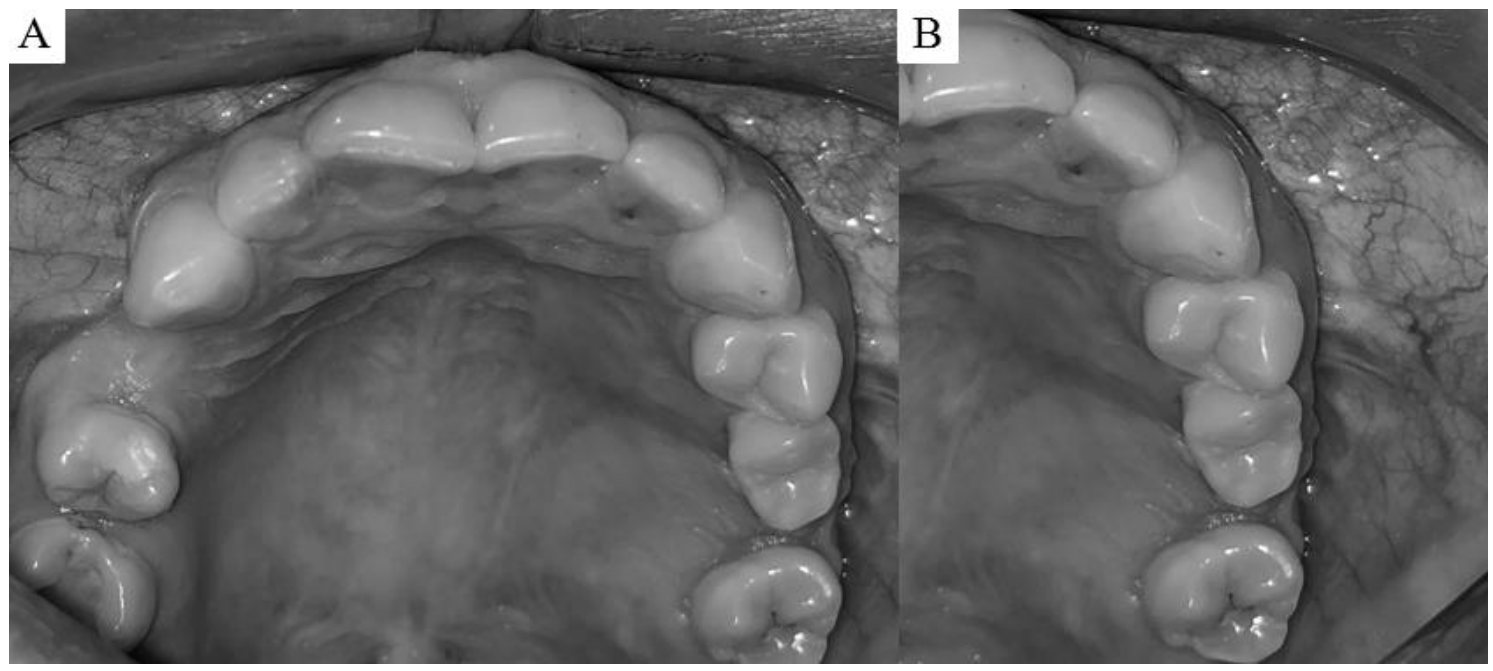

Figura 3: A- Aspecto pós-operatório de 6 meses da região do palato após cicatrização completa. B- Área de recobrimento para fechamento da fístula sem alterações de coloração ou forma da mucosa.

Durante o acompanhamento pós-operatório imediato, a paciente apresentou boa evolução, sem complicações na ferida cirúrgica e com resolução das queixas prévias. Tornou-se evidente que, as membranas de enxerto com fatores de crescimento, utilizadas como proteção da área doadora no palato, promoveram melhora na resposta inflamatória, cicatrização mais efetiva e rápida além da ausência de exposição óssea prolongada na região doadora (Figura 3). Estas características foram identificadas com aproximadamente apenas um mês de pós-operatório.

\section{Discussão}

A característica positiva da membrana de PRF é acelerar o processo de cicatrização, produzindo fatores de crescimento e leucócitos. Esses são capazes de reduzir a inflamação e infecção, diminuir o sangramento no sítio operatório devido à hemostasia vascular e estimular a recuperação de tecidos moles e duros. As vantagens obtidas através da utilização das membranas de fibrina são devido a sua liberação de fatores de crescimento importantes para uma cicatrização adequada como fator de crescimento epitelial (EGF), fator de crescimento derivado de plaquetas (PDGF), fator 
de crescimento transformador Beta (TGF-b) e fator de crescimento de fibroblastos (FGF) (AL-JUBOORI; AL-ATTAS; MAGNO FILHO, 2018)

Como a fibrina rica em plaquetas (PRF) é autogênica, não causa nenhuma reação imunológica e os fatores de crescimento em sua composição aumentam a vascularização. O PRF pode ser fácil e rapidamente preparado e implementado, é altamente biocompatível e não possui qualquer risco de infecção, uma vez que não é obtido de outros organismos vivos (AL-JUBOORI; AL-ATTAS; MAGNO FILHO, 2018)

Até hoje, vários métodos de tratamento foram introduzidos no tratamento de fístulas bucossinusais. O método mais comum e o mais antigo entre eles estava a técnica de retalho deslizante bucal (KAPUSTECKI et al, 2016). Cirurgiões orais consideram esse método como confiável, eficaz e previsível. Contudo, existem algumas desvantagens desse método, como a diminuição da profundidade do sulco vestibular, dor pós-operatória e edema (KAPUSTECKI et al, 2016). Outro método muito utilizado para defeitos maiores é o retalho palatino, que foi utilizado no presente caso, porém, como sua importante desvantagem está desnudar o palato e provocar uma cicatrização lenta e incômoda ao paciente devido a exposição óssea prolongada (VISSCHER; VAN MINNEN; BOS, 2010).

Por ser uma ótima forma de fechamento de fístulas bucossinusais, tendo quase que exclusivamente uma única desvantagem, parece adequada a escolha de utilizar o retalho palatino como fechamento das fístulas (PARAVNI et al, 2018). Com o objetivo de se evitar complicações provocadas por esta técnica, no caso proposto, foram coletadas membranas de plasma rico em fibrina para recobrimento da área palatina que se mantém cruenta após a rotação do retalho e potencializar a regeneração tecidual para o processo de cicatrização mais rapidamente.

O bom resultado observado neste caso clínico é confirmado pelos trabalhos da literatura utilizando enxertias com membranas de fibrina que, quando ativadas, liberam grande quantidade de fatores de crescimento com propriedades anti-inflamatórias e regenerativas que permitem ao organismo cicatrização mais rápida e eficiente (DEMETOGLU; OCAK; BILGE, 2018). Dessa forma, a utilização de membranas de PRF implantadas de forma aposicional sobre o palato desnudo contribui fortemente para REVISTA FLUMINENSE DE ODONTOLOGIA - ANO XXVI - N 54 - Julho / Dezembro 2020 
COPYRIGHT @ 2020 INTERNATIONAL JOURNAL OF SCIENCE DENTISTRY | AVAILABLE ONLINE http://www.periodicos.uff.br/index

uma excelente cicatrização dessa área doadora, de forma a retardar o processo inflamatório, reduzir a dor e não apresentar exposição no local. Todos esses fatores indicam que o uso do PRF em casos semelhantes a esse parece ser favorável e consideravelmente superior em relação ao uso de cimento cirúrgico comumente utilizado para recobrir a região doadora do palato. $\mathrm{O}$ presente relato de caso, se comparado com outros casos realizados no serviço de Cirurgia Oral e Maxilofacial do Hospital Federal dos Servidores do Estado do Rio de Janeiro, apresentou queixas muito menos intensas em relação ao processo inflamatório e dor relatado pela paciente. Esse fato parece estar diretamente relacionado à utilização das membranas de PRF, substituindo dessa forma o uso do cimento cirúrgico de forma satisfatória.

\section{Conclusão}

A ferida cruenta da área doadora do palato obteve uma cicatrização mais efetiva com o uso de membrana de PRF quando comparada as que cicatrizam fisiologicamente ou com o uso de cimentos cirúrgicos. A partir dessa observação, conclui-se que os fatores de crescimentos contidos nas membranas de PRF possibilitam uma cicatrização da ferida cirúrgica e uma maturação da cicatriz local de forma mais acelerada.

\section{Referências}

1. Abuabara A.,; Cortez A.L.V.; Passerin L.A.; de Moraes M.; Moreira R.W. Evaluation of different treatments for oroantral/oronasal communications: experience of 112 cases. Int. J. Oral Maxillofac. Surg. 2006; 35: 155-158.

2. Al-Juboori M.J.; Al-Attas M.A.; Magno Filho L.C. Treatment of chronic oroantral fistula with platelet-rich fibrin clot and collagen membrane: a case report. Clin Cosmet Investig Dent. 2018;10:245-249.

3. Demetoglu, U.; Ocak, H.; Bilge, S. Closure of Oroantral Communication With Plasma-Rich Fibrin Membrane. Journal of Craniofacial Surgery. 2018, 29(4), e367e370. 
COPYRIGHT @ 2020 INTERNATIONAL JOURNAL OF SCIENCE DENTISTRY | AVAILABLE ONLINE http://www.periodicos.uff.br/index

4. de Souza Lopes P.H.; de Sampaio D.O.; de Souza Menezes B.L.; do Nascimento D.F.; Torres B.C. Combined palatal flap and titanium mesh for oroantral fistula closure. Ann Maxillofac Surg. 2015;5(1):89-92.

5. Kapustecki M.; Niedzielska I.; Borgiel-Marek H.; Różanowski B. Alternative method to treat oroantral communication and fistula with autogenous bone graft and platelet rich fibrin. Med Oral Patol Oral Cir Bucal. 2016;21(5):e608-e613.

6. Parvini P.; Obreja K.; Sader R.; Becker J.; Schwarz F.; Salti L. Surgical options in oroantral fistula management: a narrative review. Int J Implant Dent. 2018;4(1):40.

7. Visscher S.H.; Van Minnen B.; Bos R.R. Closure of oroantral communications: a review of the literature. J Oral Maxillofac Surg. 2010;68:1384-1391.

8. Vyloppilli S.; Sayd S.; Thangavelu A.; Ranganathan M.; Raseel S.; Gopinath K.A. Modified Palatal Flap with a Tissue Bridge in the Closure of the Oroantral Fistulae: A Prospective Study. J Maxillofac Oral Surg. 2019 Dec;18(4):604-609. 